Kalpa Publications in Computing
Volume 2, 2017, Pages 13-19
ICRISET2017. International Conference on Re-
search and Innovations in Science, Engineer-
ing \&Technology. Selected Papers in Computing

\title{
Fixed Point Analysis of Kermack Mckendrick SIR Model
}

\author{
${ }^{1}$ Fenny J. Narsingani, ${ }^{2}$ Dr. M. B. Prajapati, ${ }^{3}$ Dr. P. H. Bhathawala \\ ${ }^{1}$ Department of Mathematics and Humanities, L. D. College Of Engineering(LDCE) \\ ${ }^{2}$ Professor, Dept. of Mathematics, Hemchandracharya North Gujarat University,Patan \\ ${ }^{3}$ Retired Professor, Dept. of Mathematics, Veer Narmad South Gujarat,University,Surat, \\ 1'fennyjn@gmail.com, ${ }^{2}$ mbpbaou@gmail.com, ${ }^{3}$ pcb1010@yahoo.com
}

\begin{abstract}
Public health is constantly under risk due to growing microorganisms. Infectious disease spread rapidly among the population in contact and so people take the different steps to reduce the transmission of disease. Compartmental model such as SIR model developed by W. Kermack and G Mckendrick are modeled for the progress of epidemic. Fixed point analysis has been applied to mathematical models of compartmental infectious disease models for understanding the long term outcome of disease. We have applied the analysis to the spread of infectious disease and obtained the threshold value and this threshold value helps us to predict when epidemic peaks.
\end{abstract}

Keywords: Reproduction number, Fixed point, Threshold value

\section{Introduction}

In 1927 Kermack and McKendrick [1] developed an epidemic model which was considered a generalized model at that time. In SIR model given by Kermack Mckendrick, the total population is assumed to be constant and divided into three classes. Susceptible class contains individuals who have no immunity to the infectious agent; any member of the susceptible class could become infected. Infectious class contains individuals who are currently infected and can transmit the infection to susceptible individuals who they contact. Recovered class contains individuals who have returned to a normal state of health after being infected and those individuals have gained permanent immunity. This model is called the SIR model [2][3]. In this model we assume that:

1. The way a person can leave the susceptible group, $\mathrm{S}$, is to become infected. The way a person can leave the infected group, I, is to recover from the disease. 
2. The recovery rate $\gamma$ and the transmission rate $\beta$ are the same for all individuals and are supposed positive

3. There is homogeneous mixing, which means that individuals of the population make contact at random and do not mix mostly in a smaller subgroup

4. The disease is novel, so no vaccination is available

5. The population size, $\mathrm{N}$, is constant and large.

6. Any recovered person in $\mathrm{R}$ has permanent immunity [5]

\section{Table 1}

\begin{tabular}{|c|l|c|}
\hline Variables & Description & Units \\
\hline $\mathrm{B}$ & Transmission rate & 1/peoplexDays \\
\hline$\gamma$ & Recovery Days & 1/Days \\
\hline $\mathrm{T}$ & Time & Peops \\
\hline $\mathrm{S}$ & Number of susceptible people \\
\hline $\mathrm{I}$ & Number of Infected people & People \\
\hline $\mathrm{R}$ & Number of Recovered people & People \\
\hline $\mathrm{N}$ & Total number of people & People \\
\hline
\end{tabular}

Given these assumptions, Kermack and McKendrick presented the following system:

$\frac{d S}{d t}=-\beta S I$

$\frac{d I}{d t}=I(\beta S-\gamma)$

$\frac{d R}{d t}=\gamma$

With the initial condition

$S(0)=S_{0}>0, I(0)=I_{0}>0, R(0)=0$

Using (1), (2) and (3) we have

$$
\begin{aligned}
& \frac{d s}{s}=-\beta I d t \\
& \frac{d s}{s}=-\frac{\beta}{\gamma} \frac{d R}{d t} d t \\
& \log S=-\frac{\beta}{\gamma} R+\log C \\
& \log \frac{S}{C}=-\frac{\beta}{\gamma} R \\
& S=C e^{-\frac{\beta}{\gamma} R}
\end{aligned}
$$

Using initial condition when $\mathrm{t}=0, \mathrm{~S}=\mathrm{S}_{0}$ on integrating equation $\frac{d s}{s}=-\beta I d t$ on both sides we get

$\log S=-\beta I t+\log C$ 


$$
\begin{aligned}
& \therefore C=S_{0} \\
& S=S_{0} e^{\frac{-\beta}{\gamma} R}
\end{aligned}
$$

The epidemic modeling of Kermack and Mckendrick SIR Model is based on Ordinary Differential Equation. An ordinary differential equation is of the form

$$
\frac{d x}{d t}=F(x, t)
$$

Subject to the condition $\mathrm{x}(0)=\mathrm{x}_{0}$

\section{Fixed Point Analysis}

Let $\mathrm{x}^{*}$ be the equilibrium point of (5)

$\mathrm{F}\left(\mathrm{x}^{*}\right)=0, \quad \frac{d}{d t} x^{*}=\mathrm{F}\left(\mathrm{x}^{*}\right)=0$

For Such equilibrium points, it is meaningful to talk about stability. For Stability we should know about trajectory that starts near a equilibrium point, stays near the equilibrium point as time increases. There are many types of stability. We consider the most common type of linear stability.

As we have an initial value $x_{0}=x^{*}+\delta x_{0}$ where $\delta x_{0}$ is small in an approximate norm

Let $x_{0} \rightarrow x(t), x^{*}+\delta x_{0} \rightarrow x^{*}+\delta x(t)$

$\therefore \frac{d}{d t} \delta x(t)=F\left(x^{*}+\delta x\right)=\left(\frac{\partial F}{\partial x}\right)_{x=x^{*}} \delta x$. The factor $\delta x(t)$ is very small, so the stability depends on Eigen values of a matrix $J^{*}=\left(\frac{d F}{d x}\right)_{x^{*}}$ consider the Ordinary differential equation $\frac{d x}{d t}=F(x)$

Where $\mathrm{x}=(\mathrm{S}, \mathrm{I}, \mathrm{R})^{\mathrm{T}}, F(x)=(-\beta S I, I(\beta S-\gamma), \gamma)^{T}$

Since the population is constant $\frac{d(S+I+R)}{d t}=0$

$S+I+R=N=$ cons $\tan t$

Therefore $I=N-S-R$

Using (4) in (6) we have

$I=N-S_{0} e^{-\frac{\beta}{\gamma} R}-R$

Substitute the value of (7) in (3) we get

$\frac{d R}{d t}=\gamma\left(N-S_{0} e^{-\frac{\beta}{\gamma} R}-R\right)$

In order to reduce the number of parameter and to make it more amenable to analysis, let us introduce a new variable

$u=\frac{\beta}{\gamma} R$ 
Since $\beta$ being an infection rate (constant) and $\gamma$ being an recovery rate(constant) and $\mathrm{R}$ has the dimension of population so $\mathrm{u}$ has the dimension of a pure number. Multiply (8) on both sides by $\frac{\beta}{\gamma}$

$\therefore \frac{d}{d t}\left(\frac{\beta}{\gamma} R\right)=\frac{\beta}{\gamma} \gamma\left[N-S_{0} e^{-u}-R\right]$

$\frac{d u}{d t}=\beta N-\beta S_{0} e^{-u}-\beta R$

$\frac{1}{\gamma} \frac{d u}{d t}=\frac{\beta N}{\gamma}-\frac{\beta S_{0}}{\gamma} e^{-u}-u$

Let us divide the (9) on both sides by $\frac{\beta S_{0}}{\gamma}$ we get $\frac{1}{\beta S_{0}} \frac{d u}{d t}=\frac{N}{S_{0}}-e^{-u}-\frac{\gamma}{\beta S_{0}} u$

Let us introduce new time variable

$d \tau=\beta S_{0} d t$

$\backslash$ Hence (9) reduces to (10)

$\frac{d u}{d \tau}=p-q u-e^{-u} \quad$ say $\quad f(u)$

Where $p=\frac{N}{S_{0}}$ and $q=\frac{\gamma}{\beta S_{0}}$, using initial condition we have

As $p=\frac{N}{S_{0}}=\frac{S_{0}+I_{0}}{S_{0}} \geq 1$ while $q=\frac{\gamma}{\beta S_{0}}>0$

As $f(u)=p-q u-e^{-u} \quad p \geq 1, q>0, u \geq 0$

To Sketch the curve it is sufficient to know the Behavior of $\mathrm{f}(\mathrm{u})$ as $u \rightarrow 0$ and $u \rightarrow \infty$ and to determine whether the curve has maxima and minima

\subsection{Behavior at 0}

We have $f(0)=p-1$ since $p \geq 1$

$\therefore f(0) \geq 0$. Let us consider the case $p>1$ then $\frac{S_{0}+I_{0}}{S_{0}}>1 \Rightarrow I_{0}>0$

This shows that there are some sick people present initially. Then $f(0)>0$

\subsection{Behavior for large $u$ values}

As $u \rightarrow \infty$ then exponential term dies away as $u \rightarrow \infty \therefore f(u) \approx-q u$

$f(u)$ becomes very large and negative and behaves like a straight line as $u \rightarrow \infty$ 


\subsection{Maxima and Minima}

Let us evaluate $f^{\prime}\left(u_{0}\right)=0$

$\Rightarrow-q+e^{-u_{0}}=0$

$\Rightarrow u_{0}=-\log q$

For $\mathrm{q}<1$ this point exist in the range $\mathrm{u}>0$ and the second derivative is $f^{\prime \prime}\left(u_{0}\right)=-e^{-u_{0}}$

This is always negative. Hence the point $u_{0}=-\log q$ if it exists is maximum

Across the $\mathrm{x}$-axis we have

$$
u \stackrel{\lim }{\rightarrow} 0 f(u)>0, u \stackrel{\lim }{\rightarrow} \infty f(u)<0
$$

$\mathrm{f}$ (u) has at most one local maximum on $\mathrm{u}>0$ thus it shows that this is a stable equilibrium point. Epidemic will be at peak if the number of infectious is maximum.

$\frac{d I}{d t}=0 \Rightarrow$ The number of infectious is at maximum

That is therefore $\frac{d^{2} R}{d t^{2}}=\gamma \frac{d I}{d t}=0$ at peak the infection stops accelerating. Since $\mathrm{R} \propto u$

$\frac{d^{2} u}{d t^{2}}=0$ at peak therefore

$\frac{d^{2} u}{d t^{2}}=\frac{d}{d t}\left(\frac{d u}{d t}\right)=\frac{d}{d t}(f(u))=f^{\prime}(u)$

$\frac{d u}{d t}=f^{\prime}(u) f(u)$

Thus at peak $f^{\prime}(u)=0($ since $f(u) \neq 0)$

Therefore we realized that only if $\mathrm{q}<1$ then the epidemic peaks

1) The number of infectious people increases if $\frac{d^{2} u}{d t^{2}}>0$

2) If $f^{\prime}(u)=0$ then at peak acceleration stops

3) $\frac{d^{2} u}{d t^{2}}=f^{\prime}(u) f(u)<0$ is always, this means the number of infectious decreases from the start.

Parameter $\mathrm{q}=\frac{\gamma}{\beta S_{0}}$ is called the threshold value. if $q<1$ then the epidemic accelerates if $q>1$ then epidemic decreases from start. 


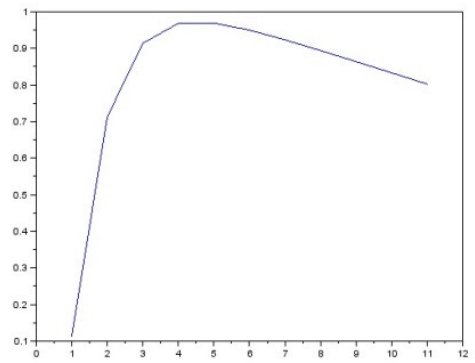

Fig. 1 Graph when $\mathrm{q}<1$

Figure 1 is the graph between the function $f(u)=p-q u-e^{-u}$ and the variable $\mathrm{u}$. From the graph it is clear that if We take $q=\frac{\gamma}{\beta S_{0}}=\frac{1}{3 *(.12) *(90)}=.03086<1$ and $p=\frac{N}{S_{0}}=\frac{100}{90}=1.1111$ then $f(u)=1.111$ $-0.03086 \mathrm{u}-\mathrm{e}^{\mathrm{u}}$,it shows that the number of infectious people increases at first and reaches at peak and then the number of infectious people start decreasing which is plotted using SCILAB.

Similarly taking $\mathrm{p}=1.111$ and $\mathrm{q}=1.23>1$ and using the equation $f(u)=1.111-1.23 \mathrm{u}-\mathrm{e}^{-\mathrm{u}}$ Using the SCILAB, we have figure 2 which is graph between $f(u)$ and $\mathrm{u}$ if $q>1$

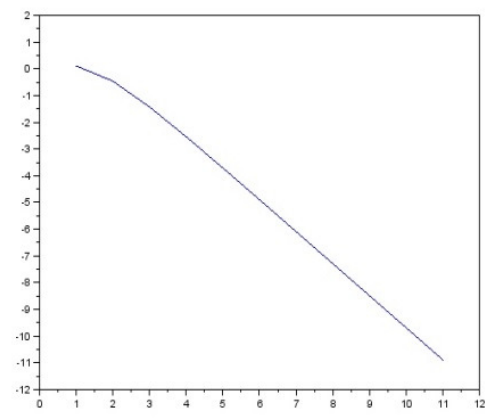

Fig:2 Graph when q >1

Graph shows that if $\mathrm{q}>1$ then the number of infectious people decreases from the he starting epidemic does not reach at the peak. This is only possible if the infectious rate $\beta$ decreases and the recovery rate increases.

\section{Conclusion}

Evolution of mathematical epidemiology into separate area of population dynamics is parallel to mathematical ecology. we have focused on the role of the threshold value (basic reproduction number) $R_{0}$,which is defined as the average number of people infected when a typical infective enters 
an entirely susceptible population. We have illustrated the significance of the threshold value $R_{0}=$ $\frac{\beta S_{0}}{\gamma}$ The disease can invade the completely susceptible people if and only if $R_{0}>1$ and epidemic dies out if $\mathrm{R}_{0}<1$.

\section{Appendix}

\section{SCILAB Code}

$$
\begin{aligned}
& \text { 1) } \quad u=(0: 1: 10) \\
& y=1.111-.03086^{*}(u)-\% \mathrm{e}^{\wedge}-(\mathrm{u}) \\
& \operatorname{plot}(\mathrm{y}) \quad(\text { for Fig-1.) } \\
& 2) \quad \mathrm{u}=(0: 1: 10) \\
& \mathrm{y}=1.1111-1.2^{*}(\mathrm{u})-\% \mathrm{e}^{\wedge}-(\mathrm{u}) \\
& \operatorname{plot}(\mathrm{y}) \quad(\text { for Fig-2. })
\end{aligned}
$$

\section{Referenes}

[1] W.O. Kermack, A. G. McKendrick, "Contributions to the mathematical theory of epidemics.I", Bull Math Biol.1991: vol.53 pp.33-55, [PubMed: 2059741],1927.

[2] W. O. Kermack, A. G. McKendrick "Contributions to the mathematical theory of epidemics II". The problem of endemicity. Proceedings of the Royal Society of London Series A, Containing Papers of a Mathematical and Physical Character: vol.138, pp. 55-83, 1932.

[3] W. O Kermack, A.G. McKendrick "Contributions to the mathematical theory of epidemics. III" Further studies of the problem of endemicity. Proceedings of the Royal Society of London Series A, Containing Papers of a Mathematical and Physical Character:vol. 141,pp.94-122,1933.

[4] Xia Ma1*,Yicang Zhou1 and Hui Cao, "Global stability of the endemic equilibrium of a discrete SIR epidemic model", vol.42,pp. 1-19, 2013.

[5] Alaa Elkadry, "Transmission rate in partial differential equation in epidemic models", Marshall university: pp. 1-17, May 2013. 